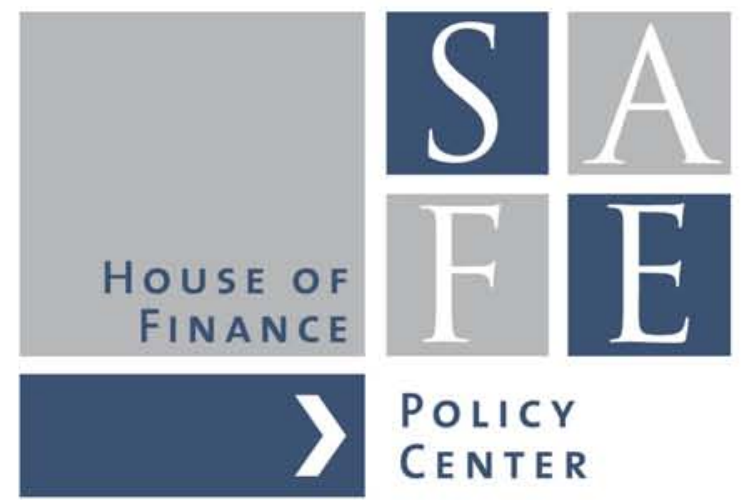

Otmar Issing

\title{
Die letzte Waffe - Helicopter Money?
}

\author{
Press Article
}

SAFE I Sustainable Architecture for Finance in Europe

A cooperation of the Center for Financial Studies and Goethe University Frankfurt 
Dieser Artikel ist bereits mit dem Titel „Die letzte Waffe - Helicopter Money?“ als Gastbeitrag in der „Börsen-Zeitung“ vom 7.02.2015 erschienen. of the Center of Excellence SAFE or its staff. 


\title{
Die letzte Waffe - Helicopter Money?
}

\author{
Otmar Issing ${ }^{1}$ \\ 7. Februar $2015^{2}$
}

Es ist noch gar nicht so lange her, da schien die monetäre Welt noch in Ordnung. Die Inflation war endlich gezähmt, Wachstum und Beschäftigung gesichert. In Amerika sprach man von der "Great Moderation“. Die Wissenschaft führte eine Debatte, was denn nun für diese traumhafte Konstellation verantwortlich sei. Die Notenbanken dank ihrer auf Geldwertstabilität gerichteten Politik galten als heißer Kandidat. Oder hatten große Teile der Welt einfach von einer Häufung glücklicher Umstände profitiert? Spätestens seit dem Ausbruch der Finanzmarktkrise 2008 herrscht jedoch ein ganz anderes Szenario. Die Geldpolitik stieß in immer mehr Ländern an den „Zero Bound“. Die Senkung der Nominalzinsen findet nun einmal bei null ihre Grenze, daran ändern auch die Versuche mit negativen Einlagenzinsen nichts Entscheidendes.

\section{Frage der Zweckmäßigkeit}

Damit war jedoch das Arsenal geldpolitischer Instrumente keineswegs erschöpft. Seit jeher steht das ganze Spektrum der Offenmarktpolitik zur Verfügung. Eine Notenbank kann durch Käufe von Vermögenswerten Geld beinahe unbegrenzt in den Verkehr bringen. Das Spektrum möglicher Aktiva reicht von festverzinslichen Wertpapieren über Devisen bis zu Aktien oder sogar Immobilien. Es ist eine Frage der Zweckmäßigkeit, von welchen Optionen die Notenbank Gebrauch machen will. So kann man beispielsweise mit Fug und Recht fragen, ob es sinnvoll ist, dass eine Notenbank (Mit)Eigentümer von Unternehmen wird.

Zahlreiche Notenbanken haben hauptsächlich private und öffentliche Schuldtitel gekauft. Diese direkt auf die Menge des ausgegebenen Zentralbankgeldes - „Quantitative Easing“ - gerichteten Maßnahmen werden oft auch als „unorthodox“ charakterisiert. Von der Sache her gehören sie zum traditionellen Instrumentenkasten der Geldpolitik - die Einordnung als „unorthodox“ trifft allerdings für den immensen Umfang der Notenbankkäufe zu.

\footnotetext{
${ }^{1}$ Otmar Issing, Präsident des Center for Financial Studies und u.a. Ex-Chefvolkswirt der Europäischen Zentralbank (EZB)

${ }^{2}$ Dieser Artikel ist am 7.2.2015 als Gastbeitrag in der Börsen-Zeitung erschienen.
} 
Weitgehend Einigkeit herrscht darüber, dass die Maßnahmen der US-Notenbank nach dem Ausbruch der Finanzmarktkrise die Gefahr eines wirtschaftlichen Zusammenbruchs in den Dimensionen der Großen Depression Anfang der dreißiger Jahre des letzten Jahrhunderts gebannt haben. Hier endet aber auch schon die Übereinstimmung. Über die Wirkungen der späteren Runden des „Quantitative Easing" wird heftig gestritten. Es spricht viel dafür, dass die weiteren massiven Ankäufe von Staatsanleihen einen vermutlich nur geringen Einfluss auf die langfristigen Zinsen gehabt haben. Das endgültige Urteil der Jury steht jedoch noch aus.

Die möglichen negativen Nebenwirkungen rücken damit umso mehr in den Vordergrund, vor allem je weniger die Maßnahmen zur Belebung der amerikanischen Wirtschaft beigetragen haben. Die amerikanische Notenbank sitzt jedenfalls inzwischen auf einem gewaltigen Berg von Wertpapieren. Es bleibt abzuwarten, welche Auswirkungen der Ausstieg aus dem Kaufprogramm oder ein möglicher Verkauf von Wertpapieren auf die Finanzmärkte und in deren Gefolge auf die Wirtschaft haben werden. Die Kombination von extrem niedrigen Zinsen und massiver Liquidität dürfte jedenfalls erneut die Anleger dazu bewegen, größere Risiken einzugehen, mit den bekannten Konsequenzen für die Vermögenspreise.

Spätestens seit die Europäische Zentralbank (EZB) ihr Ankaufprogramm für Wertpapiere bekannt gegeben hat, ist die Diskussion über die Wirksamkeit dieser Maßnahmen auch in Europa angekommen. Wegen der besonderen institutionellen Umstände des Euroraums - Kauf von Anleihen der einzelnen Nationalstaaten und des Verbots der monetären Finanzierung - reichen die möglichen Nebenwirkungen hierzulande über den rein geldpolitischen Horizont hinaus.

\section{QE ist keine Wunderwaffe}

Ein anderes spezifisches Merkmal spielt ebenfalls eine wichtige Rolle. Da nun einmal der Bankkredit im Euroraum in sehr viel höherem Maße als in den USA als Finanzierungsinstrument im Mittelpunkt steht, richtet sich das Augenmerk darauf, ob die durch den Verkauf von Wertpapieren an die EZB erhöhte Liquidität die Banken auch veranlassen wird, mehr - und hoffentlich nicht zu risikobehaftete - Kredite zu vergeben.

Weltweit hat sich das "Quantitative Easing" weder als Wunderwaffe zur Belebung der Konjunktur noch als Barriere gegen einen allenthalben - zu Recht oder zu Unrecht - als gefährlich angesehenen Rückgang der Inflationsrate erwiesen. Zu der sich ausbreitenden Skepsis trägt nicht zuletzt das Beispiel Japans bei, wo die Kombination aus jahrelangen massiven monetär finanzierten Haushaltsdefiziten alles andere als die erhofften Erfolge erzielt hat. Die Propagandisten einer solchen Politik scheinen jedoch immun gegen die Evidenz solcher Fakten zu sein. Je mehr das Vertrauen in die bisherigen Maßnahmen der Geldpolitik schwindet, desto mehr verbreitet sich die Vorstellung, dann 
müsse man eben zu „Helicopter Money“ als letztem Mittel greifen. Gemeint ist damit eine direkte Zuteilung von Geld an die Wirtschaftssubjekte, gedacht ist wohl an die privaten Haushalte.

Als Vater dieser Idee wird meist Milton Friedman genannt. Dieser hat in seinem Werk "Optimum Quantity of Money" (1969) ein hypothetisches Beispiel gebracht, in dem ein Hubschrauber Geld in einem Betrag abwirft, der genau der bereits umlaufenden Geldmenge entspricht. Unter der Annahme eines Gleichgewichts mit Vollbeschäftigung und ausgelasteten Kapazitäten führen die zusätzlichen Ausgaben der Bevölkerung lediglich zu Preissteigerungen. Am Ende des Prozesses haben sich die Preise verdoppelt, sonst hat sich nichts geändert. Diese vereinfachte Darstellung der Quantitätstheorie geht zurück auf David Hume, der schon in der ersten Hälfte des 18. Jahrhunderts ganz ähnlich argumentiert hat. Während Hume natürlich nicht an die Möglichkeit der Verteilung von Geld per Hubschrauber dachte, hat Ben Bernanke, der spätere Chairman der Fed, von dieser Möglichkeit gesprochen.

In der aktuellen Situation gilt es, zwei Fälle auseinanderzuhalten. Die Wirkungen einer Erhöhung der Zentralbankgeldmenge per Hubschrauber kann man theoretisch als äquivalent ansehen zu einer durch die Notenpresse finanzierten Steuersenkung oder Erhöhung der Transferzahlungen sowie zu auf diesem Wege finanzierten Ausgaben für Güter und Dienste.

Von diesem theoretischen Vergleich zu unterscheiden ist der Vorschlag, Geld tatsächlich auf diesem Wege per Hubschrauber oder per Scheck direkt an die privaten Haushalte zu verteilen. In einem Beitrag zur Analyse der Geldpolitik der Bank von Japan (1999) spricht Bernanke nicht nur von einem Gedankenexperiment, sondern diskutiert ernsthaft die Vorteile der direkten Verteilung von Geld. Das einzige Argument gegen diese Maßnahme läge nach seiner Ansicht in der Befürchtung der Bürger, die Regierung könnte später über eine Kopfsteuer den Betrag wieder zurückfordern. Als Folge dieser Sorge würden die Haushalte das zusätzliche Geld nicht ausgeben, sondern sparen. Es gäbe jedoch keinen Grund, diese Option ernst zu nehmen, oder anders gesagt, die Bürger würden mit dem zusätzlichen Geld tatsächlich verstärkt Güter und Dienste nachfragen. Warum also überhaupt den unsicheren Weg über das "Quantitative Easing“ gehen und nicht zusätzliches Geld, auf welchem Wege auch immer, direkt verteilen? Es geht nicht darum, dass Regierungen Schecks an die Bevölkerung verschenken. Wohltaten dieser Art in den verschiedensten Formen gehören zum Alltagsgeschäft der Politik. Die Idee des Hubschrauber-Geldes sieht jedoch die Notenbank in der Rolle des Geldverteilers. 


\section{Keine Marionette der Politik}

Die monetäre Finanzierung von Staatsausgaben - ganz abgesehen von den institutionellen Gegebenheiten des Euroraums - nimmt die Notenbank ins Schlepptau der Politik, ihre Unabhängigkeit bleibt de facto auf der Strecke. Kann man sich aber ernsthaft vorstellen, eine Notenbank könne Geld verschenken und in der Zukunft noch Herr der Geldschöpfung bleiben? Wenn dieser Sündenfall erst einmal eingetreten ist, gibt es kein Halten mehr. Tausend Gründe höchster Dringlichkeit werden die Notenbank so unter Druck setzen, dass die Notenpresse zum Selbstbedienungsinstrument der Politik degeneriert. Nicht zwangsläufig, aber doch eine absehbare Folge der Tendenz, von der Geldpolitik immer mehr und am Ende Unmögliches zu verlangen, führt zu solchen abstrusen Vorstellungen. Diese überhaupt ernsthaft zu diskutieren, ist ein weiterer Beleg für den Verfall ordnungspolitischen Denkens. Man mag sich nicht ausmalen, wohin der Weg führt, wenn sich Notenbanken auch nur auf diese Debatte einließen. 